\title{
A Research on an index of Taxi Resource Allocation in "Internet Plus" Era
}

\author{
Minghao Yan \\ School of North China Electric Power University, Baoding, China \\ 2477061602@qq.com
}

Keywords: Taxi Resource Allocation, Supply/Demand Matching, Big Data

\begin{abstract}
This article studies the extent of "Supply/Demand Matching" of the taxi resource allocation under the "internet plus" era.We set up indices, analyze supply and demand. In order to analyze the extent of "Supply/Demand Matching”, with referencing the demand-supply model of micro-economics, this article defines five major factors that are correlative to the matching-index, including time factor (applied to distinguish peak, secondary-peak, normal and low-ebb time period), distance factor (applied to distinguish different area and space), proportion of effectiveness (passenger's waiting time that is less than or equal to 15 minutes is seemed to be effective), the demand level in one certain area, the supply level in one certain area, and on the basis of above, the initial mathematical model is set up. What's more, under such background of big data, 20 groups of data were grasped to verify and correct the matching-index model. The final matching-index model will subdivide the "Supply/Demand Matching" in different space-time into five grades which are high, relatively high, medium, low and lower grade.
\end{abstract}

\section{Introduction}

In the "internet plus" era, it is not deniable that the "Double-end Subsidies" policy promoted by big companies has significantly impacted the current taxi industry, the supply-demand relationship in such new getting-a-taxi mode moves forward to a position that is not easy to be controlled, by this building new index to help companies to allocate the taxi resource becomes a key problem urgently needs to be fixed.

\section{Create and Solve Model (Set up indices, and analyze supply and demand)}

\subsection{Train of Thought for Model}

\subsubsection{Explanation on "Supply/Demand Matching”}

Under the market economy system, the unbalance of supply-demand relationship is seen an ordinary state of development, which is more obviously in taxi industry. Highlighted examples such as the too-long waiting time of passengers on the road, too-many mileages of taxi without passengers, the taxi drivers refuse to take passengers during heavy-traffic etc. indicates the unbalance of supply and demand, which is also called mismatching of supply and demand.

This article defines the "Supply/Demand Matching" as: data of taxi supply level and demand level concurrently occurs in one section of a road, and less than or equal to 15 minutes' passenger waiting time is guaranteed, regardless of peak hours or off-peak hours. It will be seen as non-effective supply/demand matching if the passenger waiting time exceeds 15 minutes, and this article defines this as supply/demand mismatching.

This article will give a reasonable determination index using the basic principles of microeconomics and referring to actual resource allocations.

\subsubsection{Mathematical Model Estimate}

Judging by taxi actual configuration and life experience, the model has close links with the following factors: the time factor (for the difference among the peak, normal and trough period), distance (used to distinguish different regions, different space), effectiveness ratio (passenger waiting time within 15 minutes is effective), the demand for a region, the supply of a region. 


\subsection{Establishment of Model I}

\subsubsection{Hypothesis of Model I}

Based on the analysis of 1.1, we believe that the model has the following relationship:

Matching coefficient index $\mathrm{k}$ and time index $\mathrm{t}$ should satisfy a proportional relationship. This article will set 4 - level for time factor, peak, sub-peak, normal, low, respectively 1,2,3,4 to represent. Once in the peak hours, matching relationship of supply and demand is less obvious, that's to say, matching index is low during the crowding period.

The index $\mathrm{k}$ and $\mathrm{l}$ is inversely proportional to the distance away from the regional city center, which shows the matching degree under different space. The more closer the city center is, the more developed road traffic is, and the greater matching index of the supply and demand.

The index $\mathrm{k}$ and effectiveness $\mathrm{p}$ is exponential correlation. This article divide effectiveness ratio into three levels: waiting 0-5 minutes for the first stage, 5-10 minutes for the second stage, 10-15 minutes for the third, more than 15 minutes for non-effectiveness ratio, which belongs to the supply and demand imbalance. Therefore, the shorter waiting time means the highest possibilities. Namely matching index is bigger.

Index $\mathrm{k}$ and quotient of $\mathrm{x}$ and $\mathrm{g}$ is exponential correlation. According to market research, we find that a region as a whole, the demand is always less than the supply, for example, in Yangpu District, Shanghai, and the number of taxis in operation always much larger than the number of passengers waiting, since the crowd will give priority to private car or subway or more convenient way to travel.

In summary, this article presents the empirical formula:

$$
\mathrm{K}=\frac{t}{\sqrt{l}} e^{p \frac{x}{g}}
$$

Wherein, K: Match indicators; t: time factor; l: regional distance from the city center; p: effectiveness ratio; $x$ : demand; g: supply.

Final match index $\mathrm{k}$ greater than or equal 0.7 means a high degree of matching supply and demand;

Final match index k greater than or equal to 0.3 , less than 0.7 means a middle degree of matching supply and demand;

Final match index k less than 0.3 means a low degree of matching supply and demand;

\subsubsection{Verification of model}

Combined with intelligent travel information and taxi market research analysis, it will verify the above formula. Take data at 13:00 on the September 7, 2015of Shanghai Yangpu District as a validation sample.

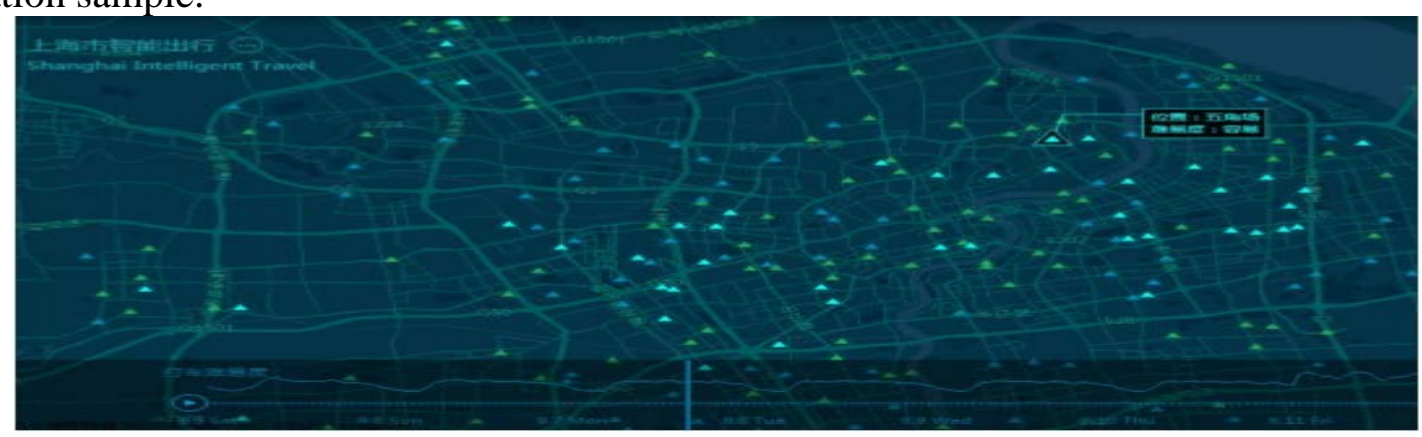

Figure 1 Taxi travel difficulty in Shanghai Yangpu District

As shown above, at this time, taking a taxi in Wujiaochang, Shanghai Yangpu District is easier. According to data obtained from reptile:

Yangpu District Demand: 2632

Supply: 1990

Substituted into the model in 1.2.1, the matching index is 0.9829 , determined in accordance with the model results, not less than 0.7 is considered as a higher degree of matching supply and demand, which corresponds to intelligent travel sites findings and results. 
In this article, data in February 18, 2015, March 3, May 9, nearly 20 sets of data, has been verified, and the results were consistent with the findings of the site and, thus, the formula has certain credibility.

\section{Summary}

This model is in the basic theory of market supply and demand as the foundation and has certain economic significance. As for the market supply and demand, the index has considerable theoretical research value and has the innovation significance, which reflects the bold use of theory, the theory of the reform of the thought.But the model is difficult to accurately determine the exact impact way of variables on the results, only to illustrate the general impact trends of various variables on the results, which makes this model still be worth improving.

\section{Reference}

[1] Zhuo Jinwu, Li Biwen, Application of MATLAB in mathematical modeling, Beijing: Beihang University press, 2014.

[2] Hal Ronald Varian, Fei Fangyu, Micro Economics: a modern perspective (Ninth Edition), Shanghai: Truth \& Wisdom Press, 2015.

[3] Nameless, Software mathematical modeling, http://www.docin.com/p-938630323.html , 2015/09/13. 